10 patients underwent a 3rd CE. In 7/10 patients with concordant initial CEs, the DY of repeat CE was 0/7. Where the 2 initial CEs disagreed, DY was 2/3.

Conclusion 1. In patients with a negative or inconclusive initial CE for IDA or OGIB, repeating the procedure has an overall DY of $25 \%(7 / 28)$.

The DY is highest when fresh blood was seen in the initial procedure $(71.4 \%)$ even if no lesions were found initially.

Patients with initially normal studies had lower DY (22.7\%).

3rd CE is only warranted by a change in presentation or discordance in the previous results, especially when one examination has identified active bleeding.

\section{PWE-104 IDA PATIENTS WITH NEGATIVE COELIAC SEROLOGY ON STRAIGHT TO TEST PATHWAY; IS D2 BIOPSY NECESSARY?}

${ }^{1}$ Faisal Shaikh*, ${ }^{1}$ Peter Mooney, ${ }^{2}$ Jason Jennings. 'Leeds Teaching Hospitals NHS Trust, Leeds, UK; ${ }^{2}$ Leeds Teaching Hospitals NHS Trust, Leeds, UK

\subsection{6/gutjnl-2018-BSGAbstracts.338}

Introduction Current BSG guidelines for Iron Deficiency Anaemia (IDA) recommend screening all adult patients for coeliac disease (CD). Duodenal biopsies are only required in patients with positive coeliac serology due to very low post-test probability for CD if serology is negative. BSG CD guidelines however recommend duodenal biopsies regardless of the serology result. We aimed to assess current practise and outcomes in the setting of a straight to test (STT) IDA pathway.

Methods We conducted a retrospective analysis of all adult patients referred on STT IDA pathway over a 3 months period. Patients who did not ultimately undergo endoscopy or had a prior diagnosis of $\mathrm{CD}$ were excluded from the study.

Results During the study period 239 patients were referred under the STT IDA pathway. Of these, 175 (male 76, female 99) underwent endoscopic investigations and were included in the study. Mean age of male and female participants was 66 and 68 years respectively. The average haemoglobin on referral was $102 \mathrm{~g} / \mathrm{L}$. Pre-endoscopy coeliac serology was only available in $44 / 175$ patients $(25.1 \%)$. Serology was positive in 1 of these patients (2.3\%)-CD was confirmed on duodenal biopsy. Duodenal biopsies were still taken in 31/43 (72.1\%) patients with negative serology, histology was normal in all cases. $110 / 131(84 \%)$ of patients without pre-endoscopy serology had duodenal biopsies taken. 9/110 (8.2\%) had abnormal duodenal biopsies. 4 cases intraepithelial lymphocytosis, 2 duodenitis and 1 Giardiasis. 2 patients had villous atrophy with suspected CD-serology came back positive in 1 patient. Second patient awaiting further investigations. There was no difference in duodenal biopsy rate based on $\mathrm{CD}$ serology availability ( $72 \%$ vs $84 \% \mathrm{p}=0.11$ ).

Conclusions Patients in STT IDA pathway with negative CD serology are unlikely to have CD. Duodenal histology is abnormal in a significant number of patients with negative serology however failure to check CD serology prior to endoscopy leads to diagnostic uncertainty and delays in diagnosis. A point of care test for CD performed in endoscopy could fill this gap. Incongruent anaemia and $\mathrm{CD}$ guidelines lead to uncertainty amongst clinicians and may explain variable practise.
PWE-105 IS THERE A CORRELATION BETWEEN SEVERITY OF BILE ACID MALABSORPTION (BAM) AND RESPONSE TO TREATMENT?

${ }^{1} \mathrm{~W}$ Siu*, ${ }^{1} \mathrm{~K}$ Ko, ${ }^{2} \mathrm{~F}$ McKiddle, ${ }^{1} \mathrm{~F}$ Clegg, ${ }^{1} \mathrm{G}$ Bain, ${ }^{1} \mathrm{~A}$ McKinlay. ${ }^{1}$ Department of Digestive Disorders, Aberdeen Royal Infirmary, Aberdeen, UK; ${ }^{2}$ Nuclear Department, Aberdeen Royal Infirmary, Aberdeen, UK

\subsection{6/gutjnl-2018-BSGAbstracts.339}

Introduction NICE guidelines in 2012 have recommended SeHCAT to be used in research in order to collect more information in its usefulness in the diagnosis and treatment of BAM. A previous study has identified a lack of consistent cutoff threshold values for abnormal SeHCAT results. The aim of this study is to determine whether there is any relationship between the severity of BAM and treatment response.

Methods Medical records of 492 patients who had a SeHCAT scan at Aberdeen Royal Infirmary between 23/7/2013 to 9/6/ 2017 were retrospectively reviewed. Mild, moderate and severe BAM were defined as $10.1 \%-15 \%, \quad 5.1 \%-10 \%$ and $<5 \%$ retention of SeHCAT after one week respectively. Data including the severity and types of BAM were recorded. Treatment responses to bile acid binders were also recorded when patients were followed up in the clinic after the scans.

Results 492 scans were performed and 51\% (252/492) of patients had abnormal SeHCAT results $(<15 \%)$ over the study period. The mean age was 51.6 with a female predominance of $69 \%(174 / 252)$.

$20 \%(50 / 252)$ of these patients had a prior diagnosis of IBS and 27\% (67/252) patients had previous cholecystectomy.

$17 \%$ (44/252) had type 1 BAM, 53\% (134/252) had type 2 BAM and 29\% (74/252) had type 3 BAM. The mean SeHCAT retention percentage was $2.59 \%$ for type 1 BAM, $7.45 \%$ for type 2 BAM and $5.63 \%$ for type 3 BAM. The difference was statistically significant $(\mathrm{p}<0.001)$.

$52 \%(132 / 252)$ of patients had treatment response documented following their scans and $13 \%$ (17/132) of these patients stopped treatment due to side effects.

For the remaining 115 patients, 71\% (12/17) of patients with mild BAM had good response to bile acid binder compared to 77\% (23/30) with moderate BAM and 78\% (55/68) with severe BAM. The difference was not statistically significant $(\mathrm{p}<0.635)$.

15 out of 90 patients who responded to colesevelam previously found cholestyramine ineffective or intolerance of it. Conclusions In our study, the mean SeHCAT retention level was significantly lower for BAM type 1 compared to BAM types 2 and 3 . There was an overall good therapeutic response to bile acid binders in patients with a positive $\mathrm{SeH}$ CAT scan. However, there was no statistically significant difference between severity of BAM and theurapeutic response.

Further prospective study using larger sample size is required to assess the accuracy and cut-offs of the SeHCAT test in diagnosing BAM as determined by theurapeutic response to BAS treatment.

\section{PWE-106 CHANGES IN THE TESTING FOR AND INCIDENCE OF COELIAC DISEASE IN THE UK 2005-2015}

${ }^{1,2}$ Joe West ${ }^{*},{ }^{1,3}$ Harmony Otete, ${ }^{4}$ Alyshah Sultan, ${ }^{1,2,5}$ Colin Crooks. ${ }^{1}$ Division of Epidemiology and Public Health, University Of Nottingham, Nottingham, UK; ${ }^{2}$ NIHR Nottingham Biomedical Research Centre, Nottingham, UK; ${ }^{3}$ School of Pharmacy, University of Nottingham, Nottingham, UK; ${ }^{4}$ Research Institute for Primary Care and Health Sciences, Keele University, Keele, UK; ${ }^{5}$ Nottingham Digestive Diseases Centre, Nottingham, UK

10.1136/gutjnl-2018-BSGAbstracts.340 
Introduction Over many previous decades the incidence of coeliac disease has been increasing almost everywhere in the world where it has been measured. However there has been a suggestion that in the last 5 years that incidence has plateaued or even declined.

Methods We used the UK Clinical Practice Research Datalink and examined the electronic health care records therein to estimate the European (2013 population) age-standardised incidence of coeliac disease 2005-2015 and the corresponding rates of serological testing (Anti-Tissue Transglutaminase (TTG) and endomysial antibody (EMA)) for the disease. We used Joinpoint analysis to examine changes in the rates of diagnosis and testing during this period.

Results There were 8177 incident cases of coeliac diseases diagnosed among 45,539,211 million person years. Over the period 2005-2015 there was an increase in age-standardised incidence from 2005 (14.6 per 100,000) until 2012 (20.3 per 100,000 ) and then a plateauing effect (figure 1, $\mathrm{p}<0.05$ ). Serological testing increased and then decreased during the same period (figure 2, $\mathrm{p}<0.05$ ).

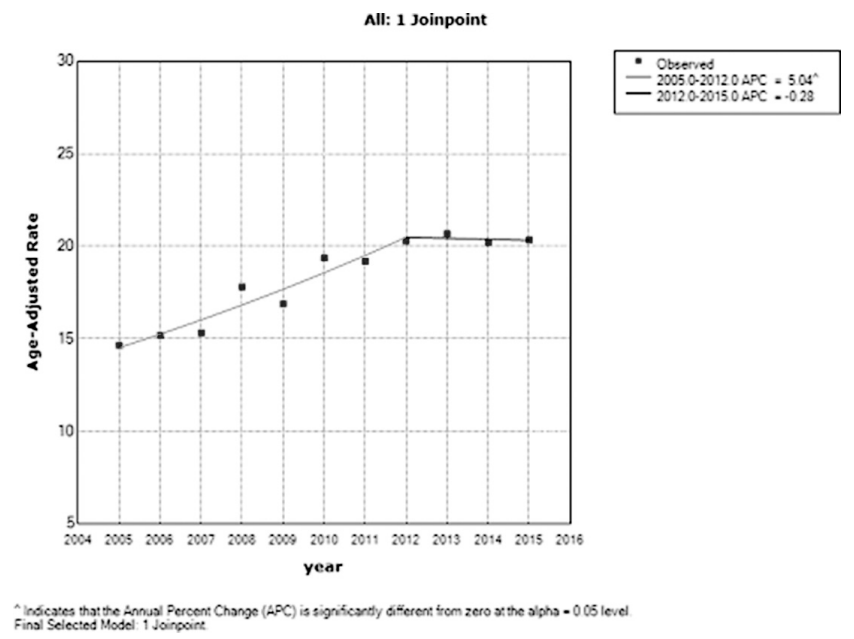

Abstract PWE-106 Figure 1 European age-standardised incidence rates of coeliac disease per 100,000 population

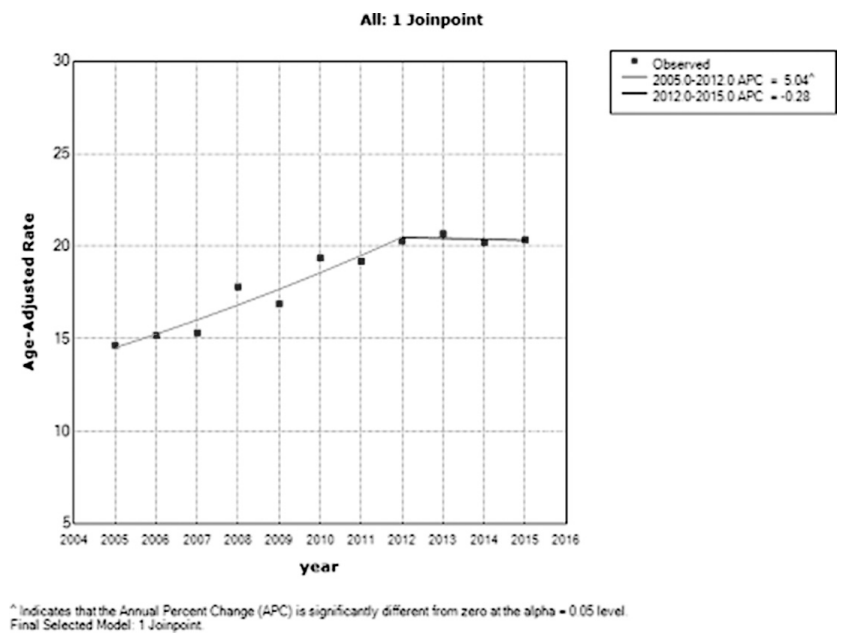

Abstract PWE-106 Figure 2 European age-standardised serological testing rates (TTG and EMA) for coeliac disease per 100,000 population
Conclusions Age-standardised rates of diagnosis of coeliac disease and serological testing have, since 2011, respectively plateaued and declined. The plateau in incidence is most likely to be a function of the corresponding decline in testing which in turn could be due to lack of resource, more targeted use of testing or that the threshold of clinically identifiable coeliac disease has been reached and a steady-state incidence rate obtained.

\section{PWE-107 FEASIBILITY OF DIRECT EUS GUIDED EMBOLISATION OF RECURRENT BLEEDING PARASTOMAL VARICES}

${ }^{1,2}$ Chander Shekhar* ${ }^{1}{ }^{1} J o a n n e$ Orourke, ${ }^{1}$ Dhiraj Tripathi, ${ }^{1}$ Colm Forde, ${ }^{1}$ Brinder Singh Mahon. 'Queen Elizabeth Hospital, Birmingham, UK; ${ }^{2}$ Manor Hospital, Walsall, UK

\subsection{6/gutjnl-2018-BSGAbstracts.341}

Introduction Recurrent bleeding from stomal varices secondary to portal hypertension can be challenging to treat. Treatments available include transjugular intrahepatic portosystemic shunt (TIPSS) and/or coil embolisation of the dominant vein passing to the stoma or surgical portosystemic shunt formation. ${ }^{1} \mathrm{~A}$ more recent approach to treat gastric varices is using endoscopic ultrasound (EUS) guidance using coil embolisation or in combination with cyanoacrylate glue. ${ }^{2}$ Endoscopic use of human thrombin in gastric varices has also been proposed as a treatment. ${ }^{3}$ To date there are no publications on the EUS guided thrombin injection combined with coil embolisation. We have experience of adopting this approach to treat stomal varices by EUS guidance

Methods We analysed data and outcomes of all EUS guided intervention for bleeding stomal varices from January 2014 to October 2017 at a regional liver transplant centre. All cases were done using Olympus EUS linear scopes, human thrombin (Tisseel; 500IU/ML) \pm coils (Nester Embolization Coils). After intubation of the stoma with the EUS scope, the dominant feeding vessel to the stoma was targeted for injection with thrombin \pm coils. All procedures were undertaken without sedation, and the majority without analgesia. Data presented as median (lower and upper quartile), unless stated otherwise. Results 19 patients (7 M and $12 \mathrm{~F}$ ) patients aged 63.5 (5470) years with recurrent bleeding from parastomal varices despite optimal medical therapy for portal hypertension had a total 27 EUS guided injections of 3000 (2500-4500) IU of human thrombin. 47\% (9/19) had thrombin alone and 53\% $(10 / 19)$ had concomitant coil embolisation. 68\% (13/19) required single intervention, 21\% (4/19) required two interventions and $11 \%(2 / 19)$ required 3 interventions with median follow up of $8(6-17)$ months, 3 lost $\mathrm{f} / \mathrm{u}$ and 3 died due to primary disease. Failure of treatment was defined as bleeding requiring transfusion or hospital admission. Only one patient failed treatment and went on to have an emergency venogram +embolisation. No immediate complications or 30 day mortality were encountered.

Conclusions EUS guided injection of thrombin \pm coil embolisation appears to be technically feasible and safe with good efficacy. To our knowledge this is the first series of EUS guided thrombin injection \pm embolisation of stomal varices. Due to the relative low number of patients and short follow up, further prospective evaluation of this promising technique is required. 